\title{
La prise en compte des troubles psychiques par l'assurance-invalidité
}

\section{Anne-Sylvie Dupont}

\section{(2) OpenEdition}

1 Journals

\section{Édition électronique}

URL : https://journals.openedition.org/rdctss/1270

DOI : $10.4000 /$ rdctss. 1270

ISSN : 2262-9815

Éditeur

Centre de droit comparé du travail et de la sécurité sociale

\section{Édition imprimée}

Date de publication : 1 avril 2020

Pagination : 218-221

ISSN : 2117-4350

\section{Référence électronique}

Anne-Sylvie Dupont, "La prise en compte des troubles psychiques par l'assurance-invalidité », Revue de droit comparé du travail et de la sécurité sociale [En ligne], 1 | 2020, mis en ligne le 01 novembre 2021, consulté le 11 novembre 2021. URL : http://journals.openedition.org/rdctss/1270 ; DOI : https:// doi.org/10.4000/rdctss. 1270

\section{(c) (†) $९$}

Revue de droit comparé du travail et de la sécurité sociale est mise à disposition selon les termes de la Licence Creative Commons Attribution - Pas d'Utilisation Commerciale - Pas de Modification 4.0 International. 


\title{
ANNE-SYLVIE DUPONT
}

\author{
UNIVERSITÉs DE NEUChÂTEL ET GENÈVE
}

\section{LA PRISE EN COMPTE DES TROUBLES PSYCHIQUES PAR L'ASSURANCE-INVALIDITÉ}

En droit suisse de la sécurité sociale, l'invalidité est définie comme une incapacité de gain de longue durée', soit comme toute diminution de l'ensemble ou d'une partie des possibilités de gain de la personne assurée sur un marché du travail présumé équilibré, étant précisé que cette diminution doit résulter d'une atteinte à sa santé physique, mentale ou psychique ${ }^{2}$. En théorie, l'assurance-invalidité helvétique offre ainsi une protection identique aux personnes atteintes de troubles mentaux et psychiques ${ }^{3}$ et aux personnes atteintes dans leur santé physique ${ }^{4}$. En pratique, les premières accèdent plus difficilement aux prestations de l'assurance-invalidité que les secondes, en particulier s'agissant des rentes. L'origine de ce phénomène n'est pas à chercher dans les textes législatifs, mais dans la jurisprudence qui s'est progressivement mise en place, depuis les années 1960 pour ce qui concerne les addictions, puis de manière plus systématique depuis le début des années 2000. Cette jurisprudence a ensuite connu une consécration dans la loi en 2008, lorsqu'il y a été précisé que l'incapacité de gain ne pouvait être prise en considération que si elle n'était pas objectivement surmontable, autrement dit si la reprise d'une activité lucrative demeurait exigible, malgré l'atteinte à la santé. Bien que cette condition s'adresse, à la première lecture, à toutes les personnes assurées - quelle que soit la nature de l'atteinte à la santé qu'elles présentent - elle pose rarement des difficultés en présence d'atteintes physiques dont le caractère limitant est attesté médicalement. II n'en va pas de même pour les troubles psychiques.

\section{I - AUPARAVANT : DES SOLUTIONS DISPARATES}

Schématiquement, avant les développements récents dont il va être question plus loin, la jurisprudence opérait une distinction entre trois catégories de troubles psychiques, l'analyse de l'invalidité obéissant à des règles différentes pour chacune. - Les addictions (médicaments, alcool, substances psychotropes) : depuis plus d'un demi-siècle, la jurisprudence considérait, de manière schématique, que ces pathologies ne pouvaient conduire à une invalidité que dans deux cas. Premièrement, il en allait ainsi seulement si ces pathologies avaient elles-mêmes causé une maladie ou un accident à l'origine d'une (autre) atteinte à la santé entravant la capacité de travail. Dans le second cas, il fallait que ces pathologies soient elles-mêmes la conséquence d'une (autre) atteinte à la santé physique ou psychique ayant valeur de maladie. Reposant sur la prémisse, davantage morale que juridique, qu'une personne dépendante était responsable de son addiction, la jurisprudence présumait que la personne assurée était capable de la surmonter. Cette

1 Art. 8 de la loi fédérale du 6 octobre 2000 sur la partie générale du droit des assurances sociales (LPGA ; RS 830.1).

2 Art. 7 LPGA.

3 M. Moser-Szeless et A.-S. Dupont (ed.), "Loi sur la partie générale des assurances sociales », Commentaire romand, Helbing Lichtenhahn Verlag, Bâle, 2018, n¹0 ad art. 6.

4 Selon l'art. 7 LPGA, la perte de gain constitutive d'invalidité (voir art. 8 LPGA) peut découler aussi bien d'une atteinte à la santé physique que d'une atteinte à la santé mentale ou psychique. 
fiction ne tenait compte ni de la gravité effective de la pathologie, ni des ressources à disposition ${ }^{5}$. - Les syndromes sans pathogenèse ni étiologie claires et sans constat de déficit organique (troubles somatoformes douloureux, fibromyalgie, fatigue chronique, etc.) $)^{6}$ : depuis la fin des années 1990, ces pathologies faisaient l'objet d'une jurisprudence très restrictive, présumant l'absence de leur caractère invalidant, cette présomption ne pouvant être renversée qu'à des conditions dont la démonstration était le plus souvent inaccessible ${ }^{7}$. - Les troubles dépressifs de gravité légère ou moyenne: depuis la mi-mai 2016, les personnes concernées par de tels troubles avaient été écartées de la protection de l'assurance-invalidité. Ces pathologies ont été présumées non invalidantes, car curables. Cette jurisprudence a été vivement critiquée, tant par la doctrine juridique que médicale.

\section{II - AUJOURD'HUI : UNE MÉTHODE D'ANALYSE UNIFIÉE}

\section{A - LA MISE EN PLACE}

La première pierre de la méthode d'analyse actuellement utilisée pour évaluer le caractère surmontable ou non d'une incapacité de travail a été posée en 2015, en lien avec les syndromes sans pathogenèse ni étiologie claire et sans constat de déficit organique. Dans un arrêt de référence, le Tribunal fédéral a jugé que l'analyse jurisprudentielle conduite jusque-là présentait le double inconvénient de ne pas tenir suffisamment compte des ressources à disposition de la personne assurée, et de dédouaner l'administration d'une instruction complète du dossier, au profit d'une analyse schématique (check list) au terme de laquelle soit la capacité de travail existe à $100 \%$, soit elle est totalement niée, sans intermédiaire possible, la seconde issue n'étant que très rarement rencontrée.

Le Tribunal fédéral a en conséquence décidé d'abandonner la présomption du caractère surmontable, au profit d'une grille d'examen structurée et normative, articulée autour de deux piliers ${ }^{8}$ :

- Le premier pilier est celui de la gravité fonctionnelle de la pathologie présentée par la personne assurée. Ce pilier se subdivise en trois axes et permet de vérifier l'empreinte du diagnostic sur l'existence de cette dernière. II s'agit de vérifier premièrement l'ampleur des limitations fonctionnelles découlant de l'atteinte à la santé compte tenu d'éventuelles comorbidités, ainsi que l'issue des thérapies entreprises, puis deuxièmement la structure de personnalité de la personne assurée, et finalement, les ressources dont elle dispose dans son environnement social.

- Le second pilier permet de vérifier la consistance des troubles en vérifiant d'une part que les limitations constatées s'étendent à tous les domaines de la vie de la personne assurée, et en mesurant d'autre part le « poids de la souffrance», traduit par le recours aux traitements et aux mesures de réadaptation disponibles.

5 Pour un exposé de cette ancienne jurisprudence, voir ATF 145 V 215 c. 4.

6 II s'agit notamment de la fibromyalgie (ATF $132 \mathrm{~V} 65$ ), des anesthésies dissociatives et atteintes sensorielles (TFA I 9/07du 9/02/2007), du syndrome de fatigue chronique et de la neurasthénie (TF 9C_662/2009 du 17/08/2010 et 9C_98/2010 du 28/04/2010), ou encore des distorsions cervicales (« coup du lapin ») sans lésions objectivées (ATF 136 V 279) et de l'hypersomnie non organique (ATF $137 \mathrm{~V} 64)$.

7 A.-S. Dupont, «Un point d'actualité en droit des assurances sociales : le trouble somatoforme (moins) douloureux? ", in S. Fuhrer (ed.), Annales SDRCA, Zurich, 2015, p. 79.

8 A.-S. Dupont, «Un point d'actualité en droit des assurances sociales : le trouble somatoforme (moins) douloureux?», op. cit., p. 82. 


\section{B - L'APPLICATION ÉTENDUE À L'ENSEMBLE DES PATHOLOGIES PSYCHIATRIQUES}

Dans une deuxième étape, le Tribunal fédéral a encore accentué la distinction qu'il faut opérer entre, d'une part le diagnostic posé et, d'autre part, les répercussions fonctionnelles de ce dernier. II a considéré qu'un simple diagnostic ne livrait encore pas d'informations suffisantes sur le caractère surmontable ou non de l'incapacité de travail, et donc sur la présence ou à l'absence d'une invalidité. Appliquée aux troubles dépressifs moyens et légers, cette réflexion a conduit le Tribunal fédéral à revenir sur sa jurisprudence et à la modifier, dans ce sens que la procédure probatoire structurée et normative mise au point pour les troubles psychogènes (supra II.A) devait désormais être appliquées dans ces cas également ${ }^{9}$. Dans la foulée, le Tribunal fédéral a retenu, en substance, qu'il n'existe pas de différence entre les pathologies psychiques lorsqu'il s'agit d'objectiver les limitations fonctionnelles qui en résultent. Les difficultés probatoires étant les mêmes, il est justifié d'utiliser dans tous les cas la procédure probatoire structurée et normative ${ }^{10}$.

\section{C - L'INCLUSION DES ADDICTIONS}

Après cette deuxième étape, restait à régler le sort des addictions qui sont également des pathologies psychiques, mais dont la pertinence pour l'obtention de prestations de l'assurance-invalidité était strictement encadrée (supra A). Dans une affaire traitée au mois de juillet 201911, le Tribunal fédéral a admis qu'il n'existait pas de raison valable, dans un régime final d'assurance sociale comme l'est l'assurance-invalidité helvétique, pour exclure d'emblée toute atteinte à la santé pertinente chez une personne qui consomme volontairement des substances psychotropes. Le Tribunal fédéral s'appuie sur la doctrine médicale, pour laquelle rien ne permet d'affirmer que persévérer dans la consommation de substances soit un phénomène volontaire, la perte de contrôle faisant en effet partie des critères diagnostiques d'une addiction. Dès lors que la personne dépendante n'est toutefois pas nécessairement privée de toute volonté face à la maladie et bien qu'elle doive mobiliser des ressources importantes pour résister à son penchant, la problématique se pose en des termes similaires pour les autres pathologies psychiques. Il s'agit là aussi de déterminer objectivement si, malgré le diagnostic, l'exercice d'une activité lucrative est ou non raisonnablement exigible.

Pour autant, la personne souffrant d'un syndrome de dépendance n'est pas dispensée de se soumettre aux traitements disponibles et exigibles. En effet, le droit suisse des assurances sociales est gouverné par le principe de l'obligation de diminuer le dommage, qui impose à la personne assurée touchée par la réalisation d'un risque social de faire tout ce qui est en son pouvoir pour peser le moins possible sur les ressources de l'assurance. A ce titre, elle peut notamment être contrainte de se soumettre à des mesures thérapeutiques, moyennant respect d'une procédure formelle par l'assureur social, sous peine de voir ses prestations réduites ou refusées ${ }^{12}$. L'articulation de cette obligation avec l'analyse de certains indicateurs de la procédure probatoire structurée, singulièrement le recours aux thérapies disponibles comme preuve de la consistance des troubles, doit encore faire l'objet de clarifications ${ }^{13}$. En revanche, le Tribunal fédéral a d'ores et déjà précisé qu'il

9 ATF 143 V 409.

10 ATF 143 V 418

11 ATF $145 \vee 215$.

12 Art. 21 al. 4 LPGA.

13 A.-S. Dupont, "La dépendance, une maladie psychique comme les autres », Analyse de l'arrêt du Tribunal fédéral 9C_724/2018, Newsletter rcassurances, septembre 2019. 
n'était plus possible - comme cela a pu l'être sous l'ancienne jurisprudence - d'ordonner des mesures d'abstinence en vue de l'instruction du dossier, singulièrement de la mise en œuvre d'une expertise médicale ${ }^{14}$.

\section{III - APPRÉCIATION}

L'unification des règles jurisprudentielles applicables aux personnes atteintes dans leur santé psychique qui sollicitent des prestations de l'assurance-invalidité est assurément une évolution positive dans la mise en œuvre du droit suisse des assurances sociales. Les différences de traitement ayant existé par le passé sont en effet difficilement justifiables d'un point de vue dogmatique, et ne peuvent être interprétées que comme l'expression de jugements de valeur qui ne sont plus défendables aujourd'hui - si tant est qu'ils l'aient été un jour, compte tenu de l'évolution de la science médicale en particulier. Cela étant, la mise en œuvre de la procédure probatoire structurée révèle que des inégalités de traitement restent possibles. Tout d'abord, le Tribunal fédéral considère que pour des motifs de proportionnalité, il est possible d'y renoncer lorsqu'elle n'est pas nécessaire, voire inappropriée ${ }^{15}$. II en va notamment ainsi en cas de majoration des plaintes ou de simulation, conclusion souvent tirée en présence d'un trouble non objectivable, même s'il a été rappelé qu'une simple tendance à l'aggravation ne suffisait pas et que les limites d'un comportement démonstratif devaient être clairement dépassées. En outre, la majoration des plaintes ne doit pas être la conséquence d'une (autre) pathologie existante ${ }^{16}$.

Par ailleurs, dans un arrêt récent qui suscite de vives réactions dans le monde médical, les juges fédéraux ont affirmé que les êtres humains étaient par principe en bonne santé et, donc, capables de travailler. En affirmant cela, ils ont non seulement relégué l'inexigibilité de l'exercice d'une activité lucrative au rang d'exception devant être appréciée strictement, mais ils ont surtout mis en avant le caractère normatif, et donc forcément abstrait, de l'exigibilité de la mise en œuvre de la capacité de travail résiduelle, en dépit des examens médicaux sur lesquels son évaluation est censée reposer.

Enfin, dans cette même affaire, le Tribunal fédéral a indiqué que les médecins devaient s'en tenir aux questions normatives formulées dans le cadre de la procédure probatoire structurée, l'administration, respectivement les juges, conservant le pouvoir d'examiner librement si les médecins ont respecté le cadre normatif, et si, sur la base de leurs constatations, l'on peut conclure à une incapacité de travail ${ }^{17}$. Bien qu'il s'en défende ${ }^{18}$, le Tribunal fédéral autorise une relecture plutôt libre des expertises médicales lorsque cellesci ne remplissent pas les exigences de la procédure probatoire structurée. II n'est donc pas très difficile de s'écarter des constatations médicales lorsque celles-ci ne conviennent (subjectivement) pas en raison de la nature du diagnostic posé. En définitive, la procédure probatoire structurée permet toujours d'écarter de l'assurance-invalidité les " mauvais» diagnostics, en minimisant l'importance de l'appréciation médicale, au profit de la lecture que l'administration, respectivement le tribunal, peut en faire. Le risque existe donc de discriminations latentes, contrairement aux discriminations avouées, et pendant bien longtemps parfaitement assumées, de l'ancienne jurisprudence.

14 TF 9C_309/2019 du 7/11/2019, c. 4.2.2.

15 T. Gächter et M. E. Meier, «Praxisänderung zu Depressionen und anderen psychischen Leiden », Jusletterch, 15 janvier 2018, $n^{\circ} 26$.

16 Voir notamment TF 9C_659/2017 du 20/09/2018.

17 ATF 144 V 50 c. 4.3 ; ATF 143 V 418 c. 6.

18 TF 9C_808/2018 du 2/12/2019, destiné à la publication ; ATF 141 V 281 c. 5.2.3. 